\title{
PIPAC (Pressurized Intraperitoneal Aerosol Chemotherapy) en el tratamiento de la carcinomatosis peritoneal. Primeros casos en Chile
}

\author{
Mario Uribe M. ${ }^{1}$, Luis Cereceda G. ${ }^{2}$, Francisco López K. ${ }^{1}$, Carlos García C. ${ }^{1}$, \\ Marco Guerrero G. ${ }^{3}$ y Victoria Vargas V. ${ }^{1}$
}

\section{PIPAC (Pressurized Intraperitoneal Aerosol Chemotherapy) in the treatment of peritoneal carcinomatosis. First cases in Chile}

\begin{abstract}
Introduction: PIPAC (Pressurized Intraperitoneal Aerosol Chemotherapy is a technique that allows laparoscopic administration of aerosol chemotherapy in the peritoneum. This procedure is utilized for treatment of carcinomatosis, for debulk abdominal tumors, increasing resectability, or for palliation of abdominal symptoms. Aim: To present the first two cases of PIPAC performed in Chile, technical considerations and review of the literature. Patients and Method: The way this program was started at Clínica Las Condes is presented. The technique is described. This procedure was performed in two females, both with refractory ascites due to carcinomatosis. Results: The procedure was uneventfully and patients were discharged 24 hours later. Both patients showed important reduction of ascites, maintained at 6 months of follow-up. Discussion: PIPAC is a safe emerging technique, with low complication rate. It is indicated in carcinomatosis of colonic and ovarian origin and in selected cases of pancreatic, bile duct and gastric carcinomatosis. More prospective, randomized studies should be done to stablish its exact role. Conclusions: PIPAC is a feasible technique to perform in our country. Preliminary results are encouraging and no complications were observed.
\end{abstract}

Key words: PIPAC; carcinomatosis; intraperitoneal chemotherapy.

\section{Resumen}

Introducción: PIPAC (Pressurized Intraperitoneal Aerosol Chemotherapy) Es una técnica que, vía laparoscopía, permite administrar quimioterapia en aerosol intraperitoneal, para el tratamiento de la carcinomatosis, ya sea para disminuir masa tumoral y aumentar la resecabilidad, o como paliación sintomática. Objetivo: Presentar los dos primeros casos de PIPAC en Chile, las consideraciones técnicas y revisión de la literatura. Pacientes y Método: Se describe la forma en que un programa PIPAC fue implementado en Clínica Las Condes. Se describe la técnica. Este procedimiento se realizó en dos pacientes, ambas portadoras de carcinomatosis con ascitis refractaria. Resultados: No hubo complicaciones. Alta a las 24 h. Ambas pacientes presentaron disminución de la ascitis, la que se ha mantenido a los seis meses de seguimiento. Discusión: PIPAC es una técnica emergente, que ha demostrado ser segura, con escasas complicaciones, cuya indicación incluye carcinomatosis por cáncer de colon y ovario y que se está extendiendo a páncreas, vía biliar y estómago. Su rol exacto está por definirse. Conclusiones: PIPAC es una técnica factible de realizar en nuestro país; sus resultados preliminares son alentadores y exentos de complicaciones.

Palabra clave: PIPAC; carcinomatosis; quimioterapia intraperitoneal.

\section{Introducción}

PIPAC (Pressurized Intraperitoneal Aerosol Chemotherapy) es una novedosa técnica, que consiste en entregar quimioterapia intraperitoneal en aerosol, a alta presión, para tratamiento de la carcinomatosis peritoneal. Sus indicaciones tienen dos objetivos fundamentales; reducir y controlar la enfermedad peritoneal hasta hacerla resecable, o un carácter paliativo, orientado al control de la ascitis refractaria, manejo del dolor, náuseas, vómitos y disminución del tamaño de masas tumorales sintomáticas.
Departamento de Cirugía. 2Departamento de Oncología. ${ }^{3}$ Departamento de Anestesiología. Clínica Las Condes. Santiago, Chile.

Recibido 2020-10-12 aceptado 21-01-22

Correspondencia a: Dr. Mario Uribe M. muribemd@hotmail.com 
Sus principales contraindicaciones son una sobrevida estimada menor de tres meses, obstrucción intestinal, pacientes con alimentación parenteral exclusiva y suturas intestinales recientes.

Las complicaciones quirúrgicas son escasas e incluyen obstrucción intestinal, hemorragia y dolor abdominal ${ }^{1,2}$.

\section{Material y Método}

\section{Implementación de un programa de PIPAC}

Para iniciar este programa, fue necesario adquirir el conocimiento teórico y asistir al Centro Hospitalario Universitario de Grenoble, Francia, donde uno de los autores (MU) pudo participar en consultas, reuniones, visitas clínicas, presenciar la realización de algunos casos y participar en 2 de ellos como primer cirujano. Lo anterior, junto a la asistencia de un experto internacional, permitió tener la certificación para realizar los primeros casos en nuestro país. Realizamos reuniones interdisciplinarias que incluyeron a cirujanos, anestesiólogos, instrumentistas quirúrgicas, oncólogos, enfermeras de pabellón, químicos farmacéuticos, personal de abastecimiento, bodega, adquisiciones, enfermeras de bioseguridad y representantes de la empresa, lo que se plasmó en un protocolo de manejo. Se obtuvieron las autorizaciones correspondientes.

\section{Consideraciones técnicas}

El pabellón debe tener los flujos de aire habituales. Debe proveerse una protección plástica del piso bajo la mesa operatoria y cubriendo al paciente, protección con mangas de plástico de todo el sistema de inyección de drogas. La máquina de anestesia debe tener alargadores, para que el anestesiólogo, pueda administrar gases y medicamentos.

Se posiciona el paciente en decúbito supino para técnica laparoscópica americana. Los trócares deben tener cuff inflables para permitir su sellado. En pacientes sin cirugías previas se hace neumoperitoneo de $12 \mathrm{~mm} \mathrm{Hg}$. en forma habitual. Se instala un trócar umbilical de $12 \mathrm{~mm}$., bajo visión directa. Se introduce otro trócar de $12 \mathrm{~mm}$, alejado del anterior. Otros trócares de $5 \mathrm{~mm}$, también con sistema de sellado, pueden ser posicionados según necesidad. Si hay cirugías previas, debe escogerse un sitio alejado de las cicatrices, accediendo al abdomen a través de una incisión de 1,5 cm, posicionando el trócar de la cámara y los otros bajo visión directa. Se toma biopsia de los implantes peritoneales, eventual citología. Idealmente no se liberan adherencias, por el alto riesgo de lesiones de asas intestinales que imposibilitarían el procedimiento. Si bien lo anterior no es una contraindicación formal, se prefiere no realizar, ya que la quimioterapia en aerosol puede acceder a toda la cavidad abdominal, incluso ante la presencia de adherencias moderadas, pero el daño a alguna asa intestinal impide realizar el PIPAC, por el altísimo riesgo de fístulas o peritonitis asociadas a la dehiscencia de suturas. En caso de encontrarse un abdomen sellado, el procedimiento no podrá realizarse. Se debe aplicar el Peritoneal Cáncer Index y registrarlo. Los trócares permiten posicionar la cámara de $10 \mathrm{~mm}$, de $30^{\circ}$ o $0^{\circ}$, y el Capnopen ${ }^{\circledR}$ (CapnomedGmbH, Villengendorf, Alemania), para inyectar la quimioterapia vaporizada a una presión de 250 psi (libras por pulgada cuadrada). Éstos deben ser fijados a un arco. La droga utilizada fue, en ambos casos, oxaliplatino $92 \mathrm{mg} / \mathrm{m}^{2}$ inyectada en 5 minutos. El procedimiento debe ser filmado, permitiendo comparaciones futuras, ya que la enfermedad peritoneal no es fácilmente cuantificable a través de los estudios imagenológicos. Se mantienen las drogas por otros 30 minutos. Cierre con técnica habitual.

\section{Precauciones}

Debe evitarse que el chorro de aerosol llegue directamente a las asas intestinales, en forma líquida y no vaporizada. Las pantallas de laparoscopía y los monitores deben estar visibles desde el exterior. La cámara debe incluir en su campo el Capnopen ${ }^{\circledR}$. El personal debe salir del recinto. La inyección se realiza remotamente. El cirujano debe controlar todo el procedimiento. Al finalizar, el personal ingresa al pabellón con sus elementos de protección, se retira el Capnopen ${ }^{\circledR}$ y los trócares. Todo se desecha de acuerdo a protocolos de manejo de drogas tóxicas.

El paciente, recuperado de la anestesia, no precisa cuidados especiales y puede reiniciar su alimentación en 4-6 h.

\section{Pacientes y resultados clínicos}

\section{Caso 1}

Mujer de 61 años, consulta en agosto de 2019 por dolor y distensión abdominal, constipación, náuseas y pirosis.

Tomografía computada revela engrosamiento parietal de aspecto neoplásico a nivel de unión del sigmoides proximal y medio, con alteración de la grasa perisigmoidea, lesiones quísticas anexiales bilaterales de aspecto neoplásico y signos de carcinomatosis. Hemoglobina: 9,6 g/dl, CEA: 60,77 ng/ ml, Ca 125: 267 U/ml y Ca 19-9: 810 U/ml. PET-CT 
compatible con lesiones hipermetabólicas en colon y anexos, engrosamiento nodular hipermetabólico peritoneal y ascitis, compatible con carcinomatosis.

En septiembre de 2019, se realiza histerectomía radical con disección pelviana abierta, omentectomía, apendicectomía y resección de ganglios metastásicos paraaórticos, sigmoidectomía con anastomosis primaria y citoreducción peritoneal. Alta el décimo día.

Biopsia: Adenocarcinoma mucinoso metastásico de origen colorrectal pT3 N2b M1, con invasión vascular linfática, venosa y perineural, budding tumoral negativo, con depósitos tumorales, implantes mucinosos en todos los órganos resecados, $\mathrm{y}$ compromiso ganglionar regional (8/16), paraaórtico e intercavoaórticos. Inmunohistoquímica del mismatch mantenido. Mutación del codón 12/13 de KRAS.

En comité oncológico se decide PIPAC, que se realiza en noviembre de 2019, sin incidentes.

Alta en buenas condiciones a las $24 \mathrm{~h}$. Evoluciona con disminución progresiva de la ascitis, con mejoría significativa de sus condiciones generales, permitiendo cirugía resectiva y quimioterapia hipertérmica intraperitoneal (HIPEC). Evolución favorable, manteniéndose en quimioterapia sistémica posoperatoria por seis meses, sin progresión de la enfermedad durante ese período.

\section{Caso 2}

Mujer de 61 años, consulta por cuadro de un año de poliartralgias, compromiso del estado general y distensión abdominal postprandial.

Tomografía computada en agosto de 2019 muestra engrosamiento parietal en tercio medio del recto asociado a masa pararrectal izquierda de aspecto neoplásico, numerosas adenopatías intraabdominales, supradiafragmáticas y múltiples localizaciones secundarias hepáticas, extensa carcinomatosis peritoneal, con ascitis y aumento de volumen anexial bilateral sugerente de enfermedad de Krukenberg y nódulos pleurales bilaterales. La colonoscopía confirma lesión neoplásica a $5 \mathrm{~cm}$ del margen anal, cuya biopsia resultó compatible con adenocarcinoma tubulopapilar moderadamente diferenciado. NRAS-BRAF y KRAS no mutado.

Se inició quimioterapia con FOLFOX y Cetu$\mathrm{ximab}^{\circledR}$, presentando ascitis a tensión, sintomática que requiere hospitalización y drenaje en múltiples oportunidades. En octubre de 2019, se demuestra progresión del compromiso peritoneal con estabilidad del resto de las lesiones. Referida para PIPAC para manejo de su ascitis. El procedimiento se rea-

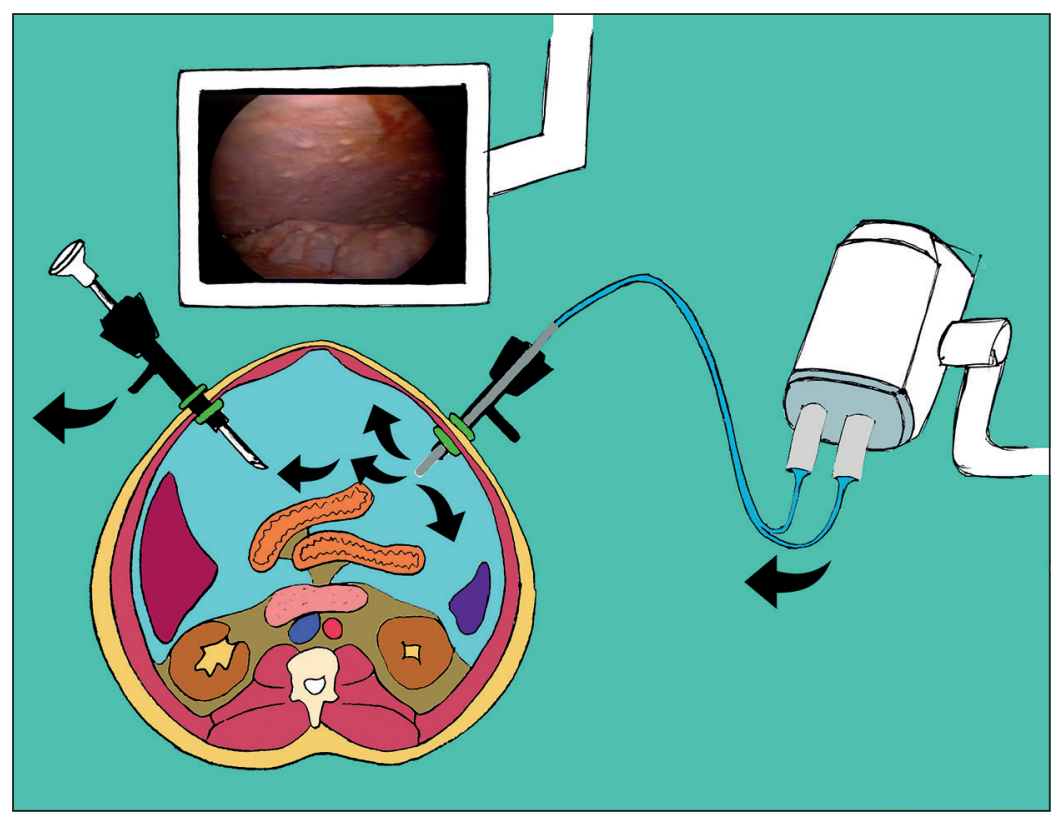

Figura 1. Administración de PIPAC (Pressurized Intraperitoneal Aerosol Chemotherapy). Se puede observar la óptica de $10 \mathrm{~mm}$ y $30^{\circ}$ y el Capnopen ${ }^{\circledR}$ por el que se inyecta, por control remoto, las drogas de quimioterapia a alta presión, la que se vaporiza en el abdomen, obteniéndose una distribución homogénea. La pantalla debe ser observada desde el exterior del pabellón.

lizó en noviembre de 2019, sin incidentes.

Alta a las $24 \mathrm{~h}$.

Disminución progresiva de su ascitis sin necesitar de punciones evacuadoras, en el seguimiento de 6 meses.

La biopsia demostró que el origen de la carcinomatosis era ovárico y no de colon, cambiándose el esquema de quimioterapia, con buena respuesta. Se mantuvo con enfermedad abdominal estable durante ese período, sin progresión extraabdominal.

\section{Discusión}

Los pacientes portadores de carcinomatosis peritoneal presentan múltiples complicaciones y baja sobrevida. La eficacia de PIPAC ha sido establecida en varias publicaciones, tanto en series prospectivas como retrospectivas. Las complicaciones quirúrgicas asociadas a PIPAC son bajas, representando alrededor de $3 \%$, porcentaje similar a la hipersensibilidad a las drogas administradas ${ }^{1}$.

PIPAC permite una distribución más uniforme y mayor penetración tisular comparado con otras formas de quimioterapia. Es bastante bien tolerado y permite a través de la laparoscopía, una adecuada evaluación de la extensión de la enfermedad perito- 
neal, posibilitando la toma de muestras para estudios histológicos y citológicos, pudiendo ser repetida sin aumentar las complicaciones asociadas a su uso ${ }^{3}$.

PIPAC ha demostrado tener una mejor relación entre la concentración en los tejidos y las dosis administradas comparado con quimioterapia sistémica, quimioterapia intraperitoneal, quimioterapia administrada laparoscópicamente e incluso que HIPEC ${ }^{4}$.

Los efectos principales de PIPAC han sido descritos en estudios experimentales, demostrando un aumento de la citotoxicidad tumoral debido a una elevación de la presión hidrostática intraabdominal, logrando una distribución homogénea demostrada con azul de metileno. Alcanza concentraciones más elevadas en los tejidos, a dosis similares. La Doxorrubicina, logra una penetración más de 10 veces mayor, comparado con HIPEC, con escasos efectos secundarios y baja toxicidad ${ }^{4}$.

PIPAC ha permitido hacer resecable hasta un $14,4 \%$ de pacientes portadores de carcinomatosis peritoneal de diferentes orígenes (mesotelioma, colorrectal, ovárico, etc.) posibilitando una cirugía resectiva a la que se le ha podido sumar HIPEC en pacientes seleccionados ${ }^{5,6}$. Se ha reportado reducción significativa de ascitis, dolor, distensión abdominal y otros síntomas gastrointestinales ${ }^{7}$. En el tratamiento de metástasis peritoneales de origen colorrectal ha destacado la negativización de los estudios citológicos para células malignas, regresión tumoral y disminución de la ascitis y una mediana de sobrevida de 27,6 meses $(10,2-47)^{8}$.

En pacientes portadores de cáncer ovárico, se obtuvo una respuesta del tumor de hasta $69 \%$, con una mediana de sobrevida de 13,7 meses. Los autores concluyen que PIPAC es posible, seguro y efectivo, con escasas complicaciones en pacientes con quimioterapia que ha sobrepasado una tercera línea9

Otras indicaciones, menos frecuentes, han sido la carcinomatosis por adenocarcinoma pancreático y de vía biliar, abriendo una nueva alternativa terapéutica para estos pacientes, demostrado ser un procedimiento seguro y bien tolerado ${ }^{10}$.

La calidad de vida mejora o se mantiene estable por periodos prolongados ${ }^{7,11,12}$. La mejoría de los síntomas abdominales se ha obtenido hasta en $64 \%{ }^{1}$.

Se han descrito algunos efectos favorables en carcinomatosis por cáncer gástrico ${ }^{3}$ aunque otros trabajos señalan que su rol en esta enfermedad está aún por determinarse ${ }^{13}$. Sus indicaciones aún pueden no estar claramente definidas, especialmente por la escasez de artículos prospectivos aleatorizados que permitan su comparación ${ }^{3}$.

PIPAC puede ser repetido siguiendo los mismos criterios de la indicación original, posibilitando un mejor control de la enfermedad y sus síntomas. No se ha descrito toxicidad relacionada con su uso reiterado y la mayoría de las series muestran un promedio de procedimientos de alrededor de 2,5 veces por paciente ${ }^{14,15}$.

Este tratamiento es estandarizado, lo que se ha obtenido gracias a registros y protocolos de calidad, aprobados por la ISSPP (International Society for the Studies of Pleura and Peritoneum) y la exigencia de los proveedores de Capnopen ${ }^{\circledR}$ de tener la certificación para la utilización correcta del dispositivo y sus condiciones de seguridad.

La evolución favorable de estos pacientes, en quienes se efectuó una cuidadosa selección, la certificación del operador, las medidas de seguridad del procedimiento y las precauciones del centro asistencial, han permitido realizar estos primeros casos en forma segura, obteniendo excelentes resultados, exentos de complicaciones.

El alto costo el Capnopen ${ }^{\circledR}$ (Aproximadamente US\$ 3.000) probablemente es un factor que dificulta su masificación. Si sus costos se hicieran más accesibles, deberán mantenerse las medidas de seguridad y la certificación de los operadores bajo los estrictos estándares actuales. Nuevos prototipos se encuentran en evaluación con resultados promisorios ${ }^{16}$. La técnica de PIPAC ha ido evolucionando paulatinamente hacia los estados IDEAL ${ }^{17}$ más confiables y seguros.

\section{Conclusiones}

PIPAC es una técnica factible de realizar en nuestro país; sus resultados preliminares son alentadores y exentos de complicaciones.

Estudios prospectivos permitirán evaluar, sus verdaderos efectos terapéuticos.

\section{Responsabilidades éticas}

Protección de personas y animales. Los autores declaran que para esta investigación no se han realizado experimentos en seres humanos ni en animales.

Confidencialidad de los datos. Los autores declaran que en este artículo no aparecen datos de pacientes.

Agradecimientos: Los autores agradecen al Dr. Julio Abba del Centro Hospitalario Universitario de Grenoble, Francia por su generosidad para enseñar la técnica y a los Dres. Gloria González y Bruno Catoia por las ilustraciones.

Conflictos de interés: no hay. 


\section{Bibliografía}

1. Alyami M, Hübner M, Grass F, Bakrin N, Villeneuve L, Laplace N, et al. Pressurised intraperitoneal aerosol chemotherapy: rationale, evidence, and potential indications. Lancet Oncol. 2019;20:e368 77.

2. Grass F, Vuagniaux A, Teixeira-Farinha H, Lehmann K, Demartines N. Hübner M. Systematic review of pressurized intraperitoneal aerosol chemotherapy for the treatment of advanced peritoneal carcinomatosis. BJS 2017;104:669-78.

3. Chia D, So J. Recent Advances in Intraperitoneal chemotherapy for gastric cancer. J Gastric Cancer 2020;20:115-26.

4. Nadiradze G, Horvath P, Sautkin Y, Archid R, Weinreich F, Königsrainer A, et al. Overcoming Drug Resistance by Taking Advantage of Physical Principles: Pressurized Intraperitoneal Aerosol Chemotherapy (PIPAC). Cancers 2020;12:34. Published online 2019 Dec 20. http://doi:10.3390/cancers12010034.

5. Alyami M, Mercier F, Siebert M, Bonnot P, Laplace N, Villeneuve L, et al. Unresectable peritoneal metastasis treated by pressurized intraperitoneal aerosol chemotherapy (PIPAC) leading to cytoreductive surgery and hyperthermic intraperitoneal chemotherapy. Eur J Surg Oncol. 2021;47:128-33. doi: 10.1016/j. ejso.2019.06.028. Epub 2019 Jun 21.

6. Ceribelli C, Debs T, Chevallier A, Piche M, Bereder J. Initial experience of pressurized intraperitoneal aerosol chemotherapy (PIPAC) in a French hyperthermic intraperitoneal chemotherapy (HIPEC) expert center. Surg Endosc. 2020;34:2803-6. https://doi. org/10.1007/s00464-020-07488-6.
7. Alonso-Gómez J, Silvestre-Rodríguez J, Bermejo-Guillén M, Artíles-Armas M, Marchena-Gómez J. Primer tratamiento PIPAC en un hospital público español. Una novedosa técnica para el tratamiento de la carcinomatosis. Cir Esp. 2019;97:177-82.

8. Bremholm S, Graversen M, Detlefsen S, Lundell L, Fristrup C, Pfeiffer P, et al. Pressurized IntraPeritoneal Aerosol Chemotherapy (PIPAC)-directed treatment of peritoneal metastasis in end-stage colo-rectal cancer patients. Pleura and Peritoneum 2020;5:20200109. Published online 2020 May 15. doi: 10.1515/pp2020-0109.

9. Tempfer C, Giger-Pabst U, Hilal Z, Dogan A, Rezniczek G. Pressurized intraperitoneal aerosol chemotherapy (PIPAC) or peritoneal carcinomatosis: systematic review of clinical and experimental evidence with special emphasis on ovarian cancer. Archives of Gynecology and Obstetrics 2018;298:24357.

10. Di Giorgio A, Sgarbura O, Rotolo $\mathrm{S}$, Schena C, Bagalà C, Inzani $\mathrm{F}$, et al. Pressurized intraperitoneal aerosol chemotherapy with cisplatin and doxorubicin or oxaliplatin for peritoneal metastasis from pancreatic adenocarcinoma and cholangiocarcinoma. Ther Adv Med Oncol. 2020;12:1-10.

11. Odendahl K, Solass W, Demtröder C, Giger-Pabst U, Zieren J, Tempfer C, et al. Quality of life of patients with end-stage peritoneal metastasis treated with Pressurized IntraPeritoneal Aerosol Chemotherapy (PIPAC). EJSO 2015;41:1379-85. Epub 2015 Jun 21. doi: 10.1016/j.ejso.2015.06.001.
12. Somashekar S, Ashwin K, Rauthan A, Rohit K. Randomized control trial comparing quality of life of patients with end-stage peritoneal metastasis treated with pressurized intraperitoneal aerosol chemotherapy (PIPAC) and intravenous chemotherapy. Pleura and Peritoneum 2018;3:20180110. doi: 10.1515/pp-20180110

13. Kumar P, Jara M, Alberto M, Rau B. The role of Pressurized IntraPeritoneal Aerosol Chemotherapy in the management of gastric cancer: A systematic review. Pleura and Peritoneum 2019;4:20180127. https:// doi.org/10.1515/pp-2018-0127.

14. Kurtz F, Struller F, Solass W, Bosmuller H, Kongsrainer H, Raymond MA. Feasibility, Safety and Efficacy of Pressurized Intraperitoneal Aerosol Chemotherapy (PIPAC) for Peritoneal Metastasis: A Registry Study. Gastroenterol Res Pract. 2018;2018:2743985. Published online 2018 Oct 24. doi: 10.1155/2018/2743985.

15. Labre V, Alyami M, Mercier F, et al. No Renal Toxicity After Repeated Treatment with Pressurized Intraperitoneal Aerosol Chemotherapy (PIPAC) in Patients with Unresectable Peritoneal Metastasis. Anticancer Res. 2018;38:6869-75. doi: 10.21873/anticanres. 13062

16. Lee H, Kim J, Lee E, Park S, Mun J, Paik $\mathrm{H}$, et al. Evaluation of a Novel Prototype for Pressurized Intraperitoneal Aerosol Chemotherapy. Cancers 2020;12:633. http://doi:10.3390/cancers12030633

17. Tate $S$, Torkington J. Pressurized intraperitoneal aerosol chemotherapy: a review of the introduction of a new surgical technology using the IDEAL framework. BJS open 2020;4:206-15. doi: 10.1002/bjs5.50257. 